\title{
Early Infantile Epileptic Encephalopathy with Burst-Suppression
}

National Cancer Institute

\section{Source}

National Cancer Institute. Early Infantile Epileptic Encephalopathy with Burst-Suppression. NCI Thesaurus. Code C116552.

A neurological disorder characterized by recurring seizures presenting within the first three months of life, progressive cerebral dysfunction, and an EEG pattern of periods of low electrical brain activity interspersed with bursts of high spiky activity. 